\title{
Design and Implementation of a Crowd Management Smart System Using Piezoelectric Sensors
}

\author{
Awad Aljuaid ${ }^{1}$, TalalAlsufyani², Ayman A. Aly ${ }^{2,3}$ \\ ${ }^{1}$ Industrial Engineering Department, College of Engineering, Taif University, PO Box 888, Taif, Saudi Arabia. \\ ${ }^{2}$ Mechanical Engineering Department, College of Engineering, Taif University, PO Box 888, Taif, Saudi Arabia. \\ ${ }^{3}$ Mechanical Engineering Department, Faculty of Engineering, Assiut University, PO Box 71516, Assiut, Egypt.
}

\begin{abstract}
Kingdom of Saudi Arabia receives a large number of pilgrims in the pilgrimage season; it is one of the places that the organizational process inside the holy palace is very difficult mission. As known crowding occurred in different places and roads, sometimes it could be dangerous for people in these places. This paper aims to implement a smart system to sense and control the crowd flow. The main goal of this system is to minimize the danger possibility from the crowded places by designing and implementing a piezoelectric material under certain selection and building criteria, to notify and warn the pedestrians about traffics in front of them. Then guide them to alternative roads to reach the same destination, and minimize the main road flow. This will minimize the number of people in the traffic areas. The system depends on the piezoelectric sensors (7BB-20-6) to sense the human flows and make a price decisions to increase the pedestrians safety in the crowding areas. The experimental results show that the proposed system is efficient in counting the humans movements as well as deciding what is the best movements to avoid accidents.
\end{abstract}

Keywords: Piezoelectric, Control unit, Smart system, sensor, PZT.

\section{INTRODUCTION}

Crowd management is concerned with coordinating crowd movement effectively - a crowd being described as a large number of people gathered into a very compact unordered body (Merriam Webster's College Dictionary, 1993). Sport and social, religious, events are part of a every society that continues to growth at home as well as abroad. The increase in popularity has resulted in larger and more diverse attendances, making crowd management and crowd control an essential and integral part of the planning process for any event. In such conditions, moving crowds creates a large amount of mechanical energy that could be used in multiple ways. Energy can be transformed by several methods, piezoelectric, electromagnetic and electrostatic transducer are one of the natural material which can transform mechanical energy to electrical energy. The techniques chosen depend on the source and its implementation. Thus according to the literature, the use of direct piezoelectric effect is one of the popular methods that fit very well with the micropower applications. In this paper, the exploratory application of piezoelectric sensor made for monitoring the crowd flow and increasing the safety. The term piezoelectricity comes from the Greek word piezoelectric meaning to squeeze or press. Piezoelectricity is developing proportional electrical charge using certain crystalline to a mechanical stress; it was discovered by J. and P. Curie in 1880 [1].In fact, they are the most commonly used materials for sensing, actuating, and transduction (energy conversion) today. Piezoelectric materials possess unique electrical characteristics that been widely used as a sensor, such as tire revolutions counter [2], Inhaler dose counter [3], Indirect piezoelectric drop counter [4]. It has many advantages: capability to transform energy, high rigidity, low cost, and different measurements. For that, the Piezoelectric ceramic sensor (PZT) have been selected as a sensor element in this paper to use the direct piezoelectric effect to sense the human crowd footsteps. The voltage generated by most PZT is relatively small and usually require amplification circuit[5]. To illustrate, a typical $27 \mathrm{~mm}$ disc of piezoelectric ceramic will increase/decrease a slightly in size in millimetres. Regardless of this small change of its thickness, PZT are used in various purposes and it can be designed in deferent specification for deferent purposes. So far, PZT made from piezoelectric ceramics the most widely used material are lead, zirconate, and titanate; because of their hight sensitivity and operating temperatures compared to other piezoelectric ceramics. The project in this paper is considered to be a smart systems. Smart systems combine several functions include the capability to sense, actuate, and control a particular condition so as to define and evaluate it. Smart systems, based on available data are efficient to expecting, determining or supporting in making decisions, thus executing smart results by the interfaces between users and systems.

To sum up, the linear correlation between electrical polarization and mechanical stress of the PZT is the feature that this paper focusing on to get and calculate the numbers and the flow rate of the moving pedestrian in a specific road.

\section{THEORY BEHIND POEZOELECTRIC MATERIALS}

The most widely known type of piezoelectric is Lead Zirconate Titanates (PZTs), combined of solutions of lead zirconate/lead titanate which are regularly doped with added elements to achieve different characteristics. The resulted ceramics are developed by adding relative quantities of lead $\mathrm{Pb}$, zirconium $\mathrm{Zr}$, and titanium oxide $\mathrm{TiO} 2$ powders, and 
apply heat between $800-1000{ }^{\circ} \mathrm{C}$, the reaction will form piezoelectric material -powder perovskite. The formed powder then mixed with a some binding material, which is shape it into the desired form. Then it experiences a paraelectric transformation to ferroelectrics shape, and the cell turn out to be tetragonal during the cooling process. As a result, it is extended in single direction and has a stable moment of dipole alignment along its c-axis. The unpoled ceramic is made up of various arbitrarily directed fields and so has no net polarisation. Appling a high electric field will align unit cells as nearly as possible to the field being applied; in a method called poling, which gives the ceramic a stable polarization. In this case, the material exhibits both the straight and opposite piezoelectric effects [6]. Piezoelectric sensor is defined as a deformation of the piezoelectric material which yields a relative difference in the electrical polarization of piezoelectric material, i.e. the charges shows on other sides of the piezoelectric material when loaded. The resulted piezoelectric outcome can be defined as:

$$
\Delta Q=k \cdot \Delta F
$$

Where $\Delta Q=$ changes the load on some other sides of piezoelectric materials; $\Delta F=$ changes the load on the other sides; and $k=$ proportionality factor set on the sensitivity of the sensor.

As mentioned previously the electrical charge is relatively small and unstable, hence the need to use charge amplifier to find the electrical charge accurately and with accuracy. A usual amplifier structure is indicated in Fig.1.

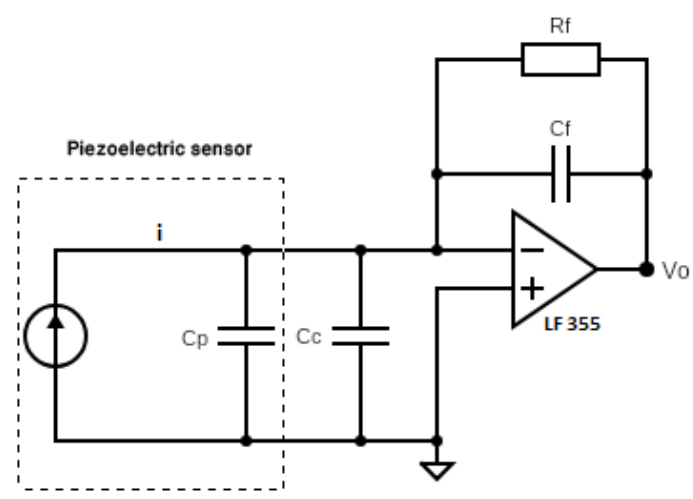

Fig. 1.Typical piezoelectric sensor charge amplifier structure

The amplifier in general

$$
\Delta U_{\text {out }}=\frac{\Delta Q}{C_{f}}
$$

In which $C_{f}=$ feedback capacitor

Sub Eq. (1) in Eq. (2):

$$
\Delta U_{\text {out }}=\frac{k \cdot \Delta F}{C_{f}}
$$

As shown From the Eqs. 2 and 3, it is obvious that the result of the amplifier is relative to the electrical charge which is resulted from the sensor.
Then as the difference in the electrical charge provided by the sensor is relative to the difference in the mechanical stress, on that account the change in the amplifier's output voltage is relative to the mechanical stress variation.

In order to produce certain amount of voltage , force is required, thus when applying a force a small amount of deflection will occur, voltage produced relatively with the deflection. Piezoelectric could be represented as a electrical charge producer $\mathrm{q}$ in corresponding to a capacitance $C_{N}$, fig.2 shows the full Piezoelectric crystal circuit as a charge generator.

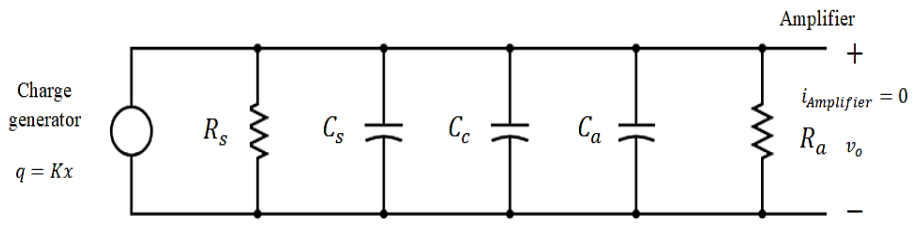

Fig. 2. Piezoelectric crystal as a charge generator

Where is $\mathrm{Rs}=$ crystal Resistance, $\mathrm{Cs}=$ Crystal capacitance, $\mathrm{Cc}=$ Cable capacitance, $\mathrm{Ca}=$ Amplifier capacitance, and $\mathrm{Ra}=$ Amplifier resistance, In order to simplify the charge generator in fig. 2 for simulation purposes, the equivalent circuit can be represented as shown in fig.3.

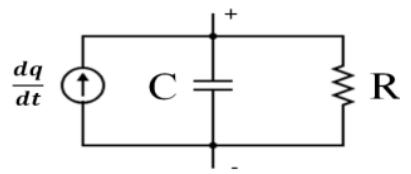

Fig.3. Equivalent circuit of the piezoelectric charge generator Where is $\mathrm{C}=\mathrm{Cs}+\mathrm{Cc}+\mathrm{Ca}$ and $\mathrm{R}=\mathrm{Rs} \| \mathrm{Ra}$, thus

$$
V_{o}=i R=\left(\frac{d q}{d t}-C \frac{d V_{o}}{d t}\right) R
$$

Or

$$
R C \frac{d V_{o}}{d t}+V_{o}=R \frac{d q}{d t}
$$

After applying Laplace Transformation

$$
\frac{V_{o}(s)}{q(s)}=\frac{R s}{R C s+1}
$$

In order to reach the desired simulate results it is required to changing the input to $\mathrm{F}(\mathrm{s})$, thus by knowing that $q=d F$ the rearranged transfer function is given as follows

$$
\begin{aligned}
& \frac{V_{o}(s)}{F(s)}=\frac{d R s}{R C s+1} \\
& \frac{V_{o}(s)}{F(s)}=\frac{\left(\frac{d}{C}\right) R C s}{R C s+1}
\end{aligned}
$$

Then taking $t_{f}=R_{f} C_{f}$ After applying the amplifier circuit mentioned in fig. 1

$$
\frac{V_{o}(s)}{F(s)}=\frac{-\left(\frac{d}{c_{f}}\right) t_{f} s}{\left(t_{f} s+1\right)}
$$




\section{SYSTEM PROTOTYPE}

The system uses as mentioned Piezoelectric sensors in order to calculate the pedestrians number passing through a given area, thus based on that and on specific algorithms it is possible to calculate the walking humans flow rate as well as approximate number of humans in the desired area, Because of this, precautionary measures can be automatically such as alerting and redirecting the flow of pedestrians to another path. The system uses colour code and sound buzzers in addition to full gate control to alert the new coming pedestrian about possibility of overcrowding in front a head. System block and Functional diagram is represented in fig.6 .

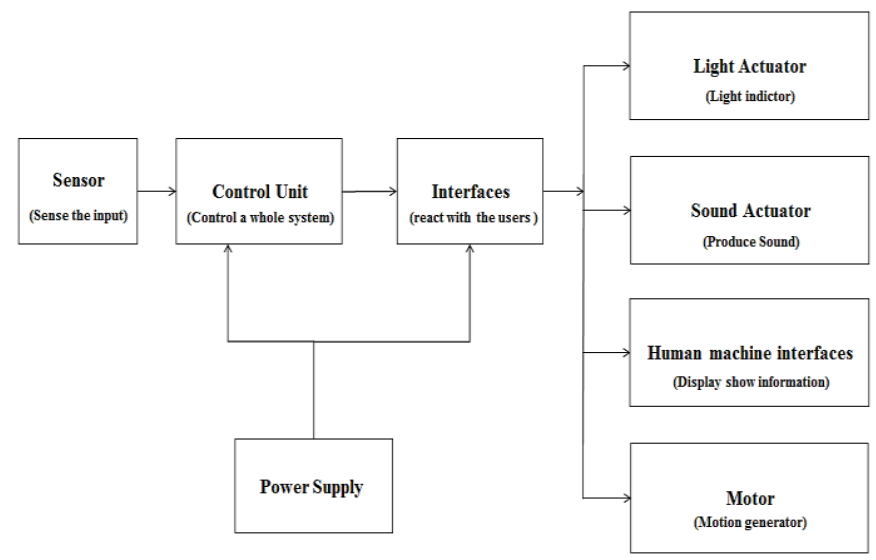

Fig.4. System block and Functional diagram representation

\subsection{Footstep Piezoelectric Sensors Structure}

This sensor was developed by joining two square sheets of rubber padded by a pieces of piezoelectric plate, as shown in Fig. 5(a) and Fig. 5(b).Rubber materials either its natural or synthetic is known to be a shock absorber, Hence can absorb part of the force applied by the footsteps ,thus using double layers of rubber sheets in the square piece covering the piezoelectric sensors from both sides is required to prevent breaking or fracture. The initial concept is to design a series of piezoelectric sensors with a length equal to the width of the road in which three square parts of $30 \mathrm{~cm} \times 30 \mathrm{~cm}$ dimensions are found in each meter range. The square rubber pieces length is built to be $5 \mathrm{~cm}$ longer than the average human foot length [7], to ensure that almost every move of the foot hits the sensor belt, every square rubber piece is connected to five piezoelectric sensors at the corners and in the center.

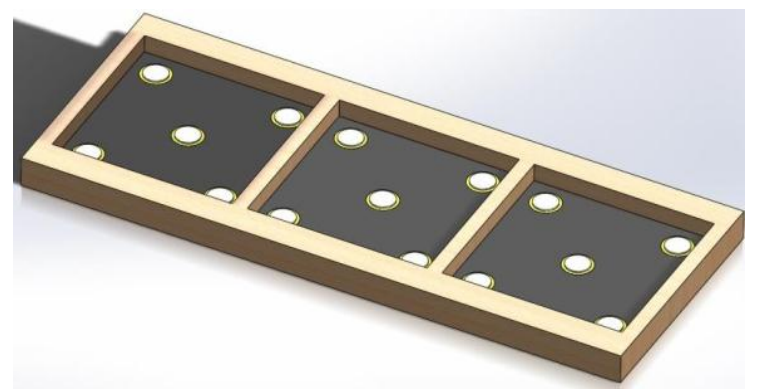

Fig. 5(a) Theoretical design of therubber sheet with piezoelectric sensors located on it

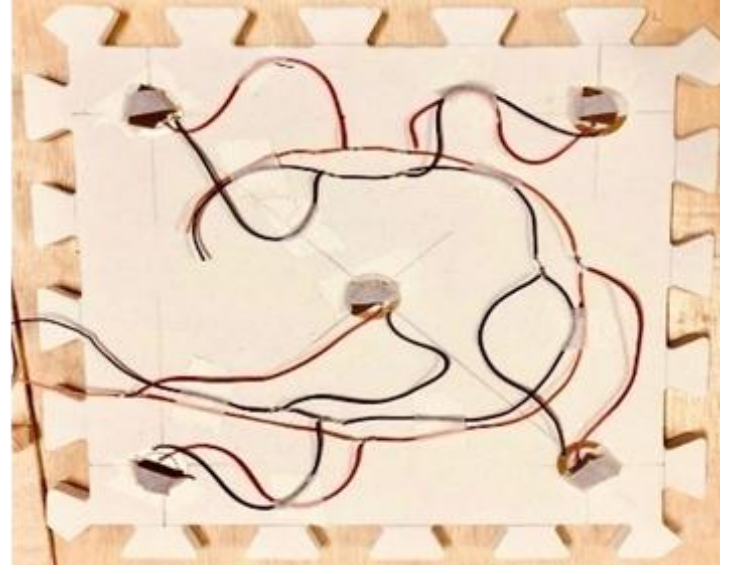

Fig. 5(b) Acctual rubber sheet with piezoelectric sensors

The prototype firstly was made of wood sheets and it was noted that it was not able to withstand the average human weights, as a consequence it was decided to use foam floor rubber materials with thickness of $5 \mathrm{~mm}$. The chosen material is called foam rubber flooring and EVA flooring, Foam rubber which has been made from a foaming material to make an air-filled matrix material. Foam rubbers are usually made from either natural latex or polyurethane, thus better protection for piezoelectric sensors in addition to increase the sensors durability. Fig.6(a) and Fig.6(b) shows the full theoretical as well as the actual mechanical structure of the desired piezoelectric sensor belt.

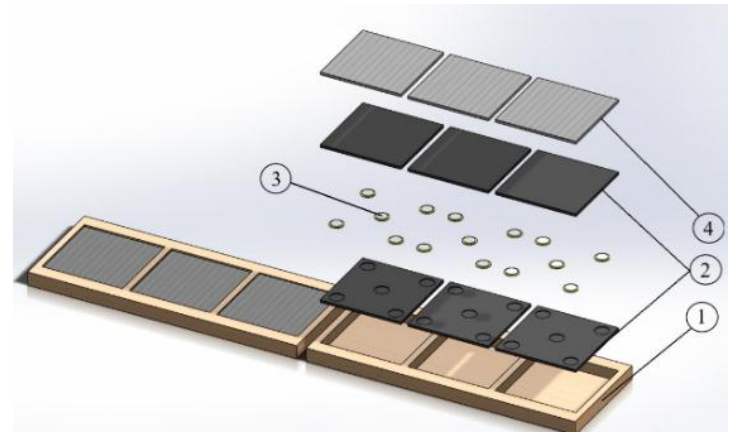

Fig. 6(a) Theoretical mechanical structure of the desired piezoelectric sensor belt

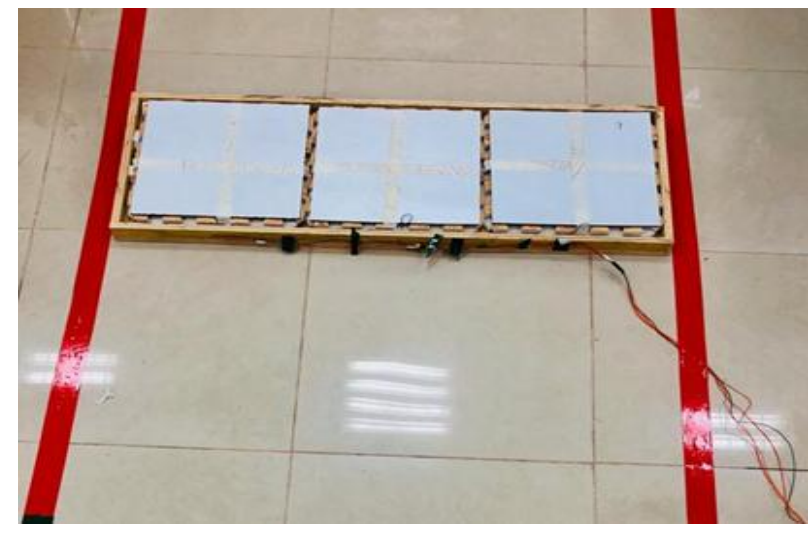

Fig. 6(b) Theoretical mechanical structure of the desired piezoelectric sensor belt 
The main Subsystems and Components the typical modern system can be divided into the following subsystems and Components.

\subsection{Sensors Selection}

The sensor selected for the experiment mentioned in this paper is the piezoelectric actuator disc with the model number (7BB-20-6), the selection of this sensor done due to its design criteria, availability, total cost, the ease of installation, and the other specifications mentioned in Table 1, to detect and locate possible overcrowded areas by generating electricity for every footstep.
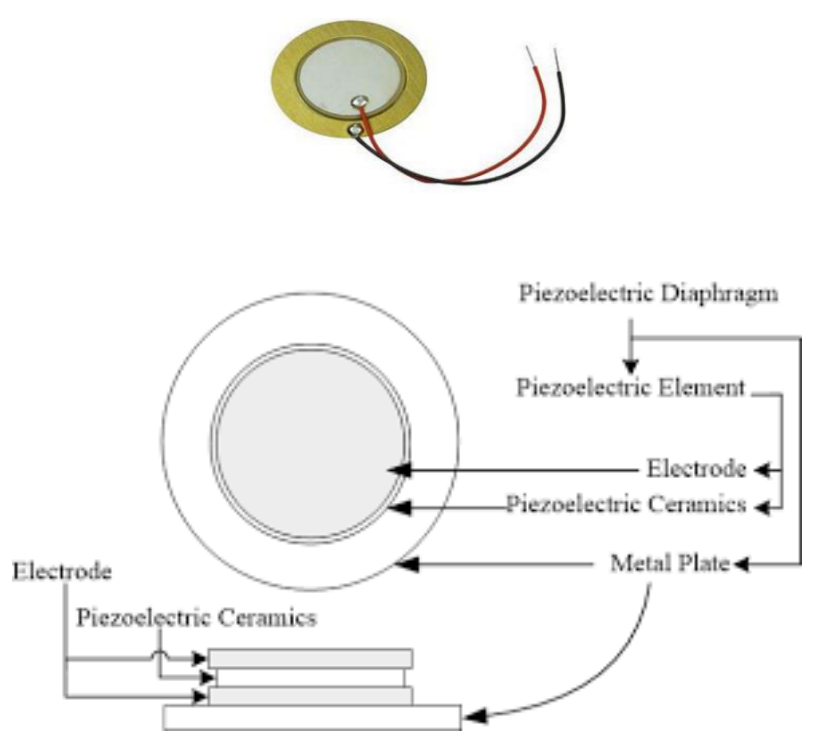

Fig.7 Piezoelectric sensor selected for the experiment (7BB20-6) with its layout discretion

Table 1. Specific parameters of the sensor selected the experiment [8]

\begin{tabular}{|l|c|}
\hline Plate size diameter, $\mathrm{D}(\mathrm{mm})$ & 20.0 \\
\hline Element size diameter, $\mathrm{d}(\mathrm{mm})$ & 14.0 \\
\hline Electrode size diameter, $\mathrm{b}(\mathrm{mm})$ & 12.8 \\
\hline Thickness, $\mathrm{T}(\mathrm{mm})$ & 0.42 \\
\hline Metal plate thickness, $\mathrm{t}(\mathrm{mm})$ & 0.20 \\
\hline Resonance frequency, fr $(\mathrm{kHz})$ & $6.3 \pm 0.6$ \\
\hline Capacitance, $\mathrm{C}(\mathrm{nf})$ & $10 \pm 30 \%$ \\
\hline Dielectric Constant, $\mathrm{Er}$ & 1510 \\
\hline Resonant Impedance, $\mathrm{R}(\Omega)$ & $\leq 300$ \\
\hline Jnput Voltage, $\mathrm{V}$ max $(\mathrm{V})$ & 30 \\
\hline Operating temperature, $\left({ }^{\circ} \mathrm{C}\right)$ & $-20 \sim 70$ \\
\hline
\end{tabular}

\subsection{Alternative Roads/Paths to Redirection The Pedestrian Motion}

The piezoelectric sensors belt is placed within certain distance from the alternative roads/paths, that is used for redirecting the pedestrian motion to that path as shown in fig. 8 in case of overcrowding, and reduce the possible resulting risks. to illustrate, the smart system detects that the number of passing over series piezoelectric sensors, is equal to or bigger that the critical number of pedestrian, the system will directly alert and notify all pedestrians about the possible danger, using sound and light signal, as well as informative boards. However in case the number of pedestrian kept passing critical number of pedestrian the system will open the gates to the pre-designed alternative roads . Time is very important variable in these situations, and has been taking in consideration, so that if the pedestrian take the secondary road, then by the time they reach the end of the road, they will not fall in overcrowding areas again.

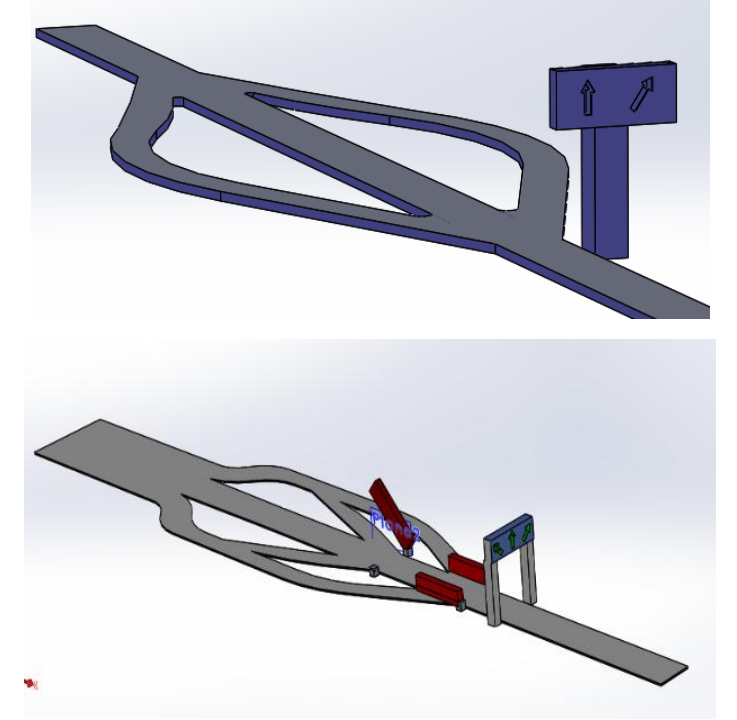

Fig.8 The initial structure proposal for the pedestrian Alternative roads/paths

\subsection{Control Unit}

A control unit for processing data and making decisions is needed in the presented document, and various types of control units exist: PLC, Microcontroller, Microcomputer and Computer. Selection of the Control Unit was made according to the design criteria. Thus microcontrollers are the most subtle control unit for this project. In order to meet the project selection criteria ATtmega 328 chip provided in Arduino Uno is chosen as a control unit to control and actuate the electric signal coming from the PZT.

\subsection{Actuator Selection}

There are several control algorithms could be applied for certain conditions. Control algorithms of complex systems can be easily utilized by using digital signal processing. The different Control algorithm types including: on-off , and continues (PID, artificial ) feed forward. Because all the components are of an on-off type, all of it can operate this system in a different approach, where is the best decision is to use on-off event driven control loop. where all the algorithm had been written and tested on the prototype control chip Atmega 328. 


\subsection{Human Machine Interface Subsystem}

In order for the project to alert pedestrian about the crowded area in the front, the project required an Actuators to complete the desired task for the crowd management system. Actuator as a definition is a device that converts the incoming electrical energy into light energy such as LED or kinetic energy such as the motor, pump and turbine..etc. initially Light and gate motor have been chosen for the system to interact directly with pedestrians in the system installation area or roads, fig.9 represent the prototype light sign with direction arrows to alert and guide the users or pedestrians to safety.
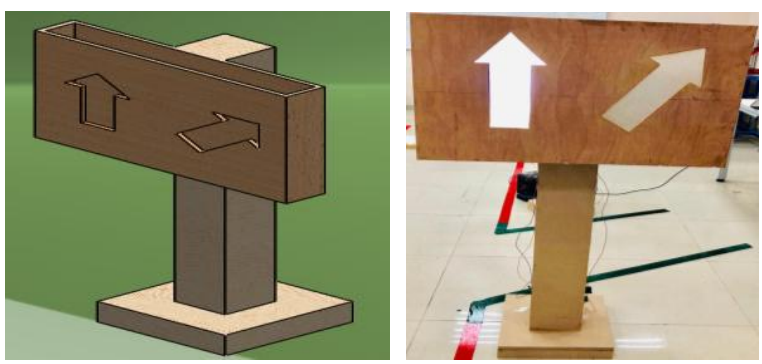

Fig.9 The theoretical and actual designed of the prototype light guiding sign

\subsection{Human Machine Interface Subsystem}

The human machine interfaces is an interface that connects users/customers to the systems. The inanition is to use the interface to communicate with the microcontroller and sensors to output information and display it to users and pedestrian and even all the specific information to the crowd management authority, with taking in concentrations that the sign must be viewable for clear information, not complex and comfortable for eyes.

\section{SIMULATION FOR DC MOTOR CHARACTERISTICS}

\subsection{Modelling of Motor Dynamics}

As mentioned in this paper there are electromechanical gates for the alternative roads will be controlled fully by the system algorithm with an emergency option to control it manually. A modeling of motor dynamics has been done for an arm rotational gate, starting by the electrical characteristics of the motor as fallows

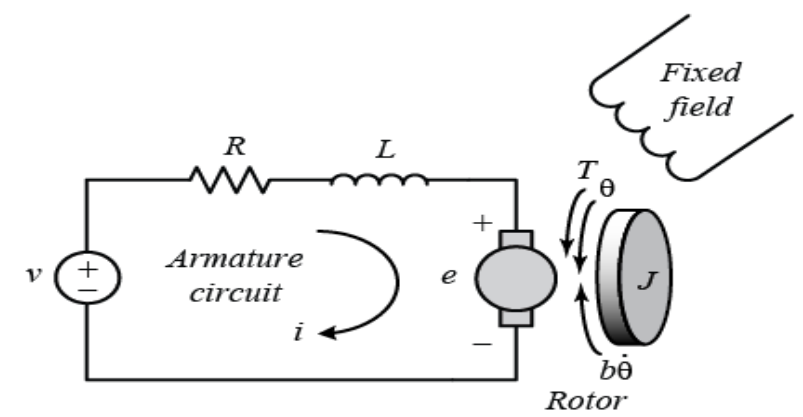

Fig. 10 A prototype of an pedestrian sign with light Actuator
Based on Newton's law with Kirchhoff's law, the mathematical differential equation defining electrical elements of the controlled motor can be obtained, the electrical correspondent of the armature circuit, can be defined as an inductance $\mathrm{L}$ in series with a resistance $\mathrm{R}$ in series with EMF voltage which resists the source of voltage. By applying Kirchhoff's law across the electrical loop by adding voltages through R-L circuit gives:

$$
\sum V=V_{i n}-V_{R}-V_{L}-V_{e m f}
$$

Then, substituting and simplifying with Ohm's law :

$$
\begin{gathered}
V_{\text {in }}=V_{R}+V_{L}+V_{\text {emf }} \\
V_{\text {in }}=R_{a} i(t)+L_{a} \frac{d i_{a}(t)}{d t}+K_{b} \omega \\
V i n-K_{b} \omega=R_{a} i(t)+L_{a} \frac{d i_{a}(t)}{d t}
\end{gathered}
$$

Simplifying with Laplace transform gives:

$$
\begin{gathered}
V_{\text {in }}(s)-K_{b} \omega(s)=R_{a} I(s)+L_{a} s I(s) \\
V_{\text {in }}(s)-K_{b} \omega(s)=\left(R_{a}+L_{a} s\right) I(s) \\
I(s)=\frac{V_{\text {in }}(s)-K_{b} \omega(s)}{\left(R_{a}+L_{a} s\right)}
\end{gathered}
$$

The motor developing a torque, yields an angular velocity $\omega=$ $\mathrm{d} \theta / \mathrm{dt}$, consistent with the inertia $\mathrm{J}$ and damping friction $\mathrm{B}$, of the motor and load.

Mathematical differential equations of the energy balance on the motor system by:

$$
T_{m}=T_{M}+T \omega+T_{a}+T_{l}
$$

Substituting the following values: $T_{m}=K_{t} i, T_{M}=J \frac{d^{2} \theta(t)}{d t}$, $T_{\omega}=B \frac{d \theta(t)}{d t}$, and $T_{a}=k \theta(t)$

$$
T_{m}=J_{m} \frac{d^{2} \theta(t)}{d t}+B_{m} \frac{d \theta(t)}{d t}+k \theta(t)+T_{l}
$$

Taking Laplace transform and rearranging, gives:

$$
\begin{gathered}
T_{m}(s)=J_{m} s^{2} \theta(s)+B_{m} s \theta(s)+k \theta(s)+T_{l}(s) \\
T_{m}(s)-T_{l}(s)=\left(J_{m} s^{2}+B_{m} s+k\right) \theta(s) \\
K_{t} I(s)-T_{l}(s)=\left(J_{m} s^{2}+B_{m} s+k\right) \theta(s) \\
\frac{\theta(s)}{K_{t} I(s)-T_{l}(s)}=\frac{1}{J s^{2}+B s+k}
\end{gathered}
$$

The electrical and mechanical motor components are combined through the torque equation ,the motor open loop transfer function without input load voltage Vin(s), ad shaft 
angle $\theta(\mathrm{s})$, given by:

$$
\begin{aligned}
& G(s)=\frac{\theta(s)}{V_{\text {in }}(s)} \\
& =\frac{K_{t}}{L_{a} J_{m} s^{3}+\left(R_{a} J_{m}+B_{m} L_{a}\right) s^{2}+\left(R_{a} B_{m}+K_{t} K_{b}\right) s}
\end{aligned}
$$

The following motor characteristics fig. 11 is used in this project prototype to test the algorithm, however the simulation in fig. represent the testing prototype to ensure that the system, algorithm and components is working and fully functional, thus better motor with batter characteristic is considered in full prototype or in further developments.

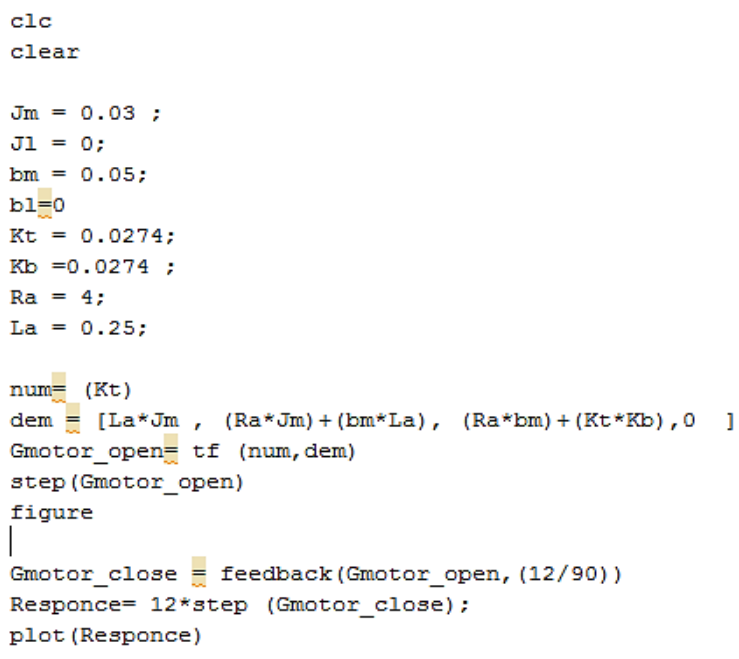

Fig.11 Motor characteristics used in the simulation in m-script After adding a sensor to obtain 90 degree angle, and by using step function In Matlab as shown in fig. 12 we got the desired output, but it takes 130 second to open the gate according the gate motor barometers see fig. 15 ,However the chosen gate motor is changeable according the installation area, where it's optional to reduce the gate opening time by choosing a motor works perfectly with the desired area.

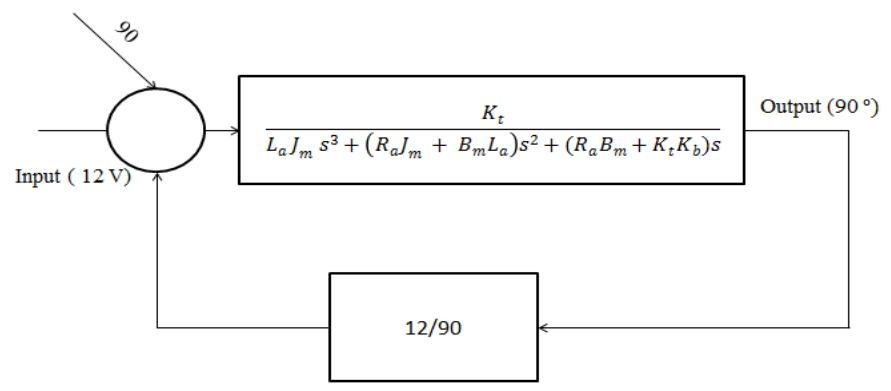

Fig.12 The Motor transfer function in block diagram

\section{RESULTS AND DISCUSSION}

\subsection{Sensors testing values}

The fallows figures fig.13,fig.14 displays the values of the sensors voltage before, during, and after applying loads on it, In addition to the number of pedestrian passing through, keep in mind that the voltage values in fig. 13 is amplified by the amplifying circuit mentioned earlier in fig.1. Voltage and human weight has a Positive relationship in such a manner that if the weight increases the voltage will increase, these results concludes that this project.

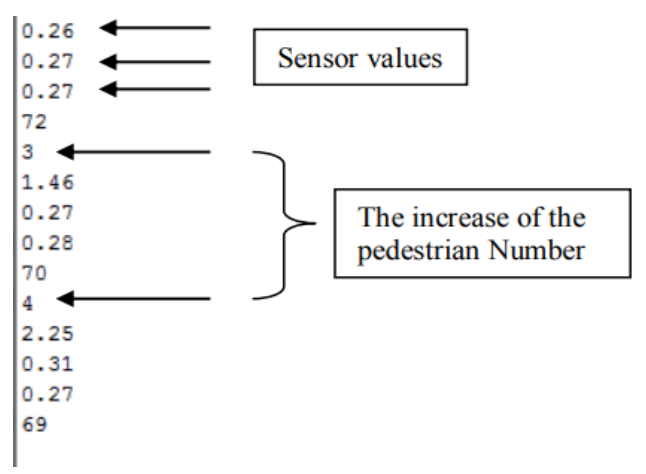

Fig.13 Analog signals of the three sensors values with the counting number of the moving pedestrian

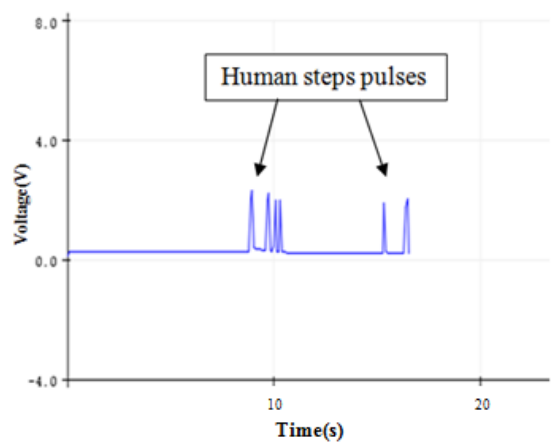

Fig. 13 Actual footsteps pulse signals on the piezoelectric sensors

\subsection{Motor Actuator}

The parameters that been used to model the gate motor performance in this study listed fig. 10 have been used in the transfer function equation (22), and resulted with the graph below in fig.15. Note that the horizontal axis represent the time in seconds, and the vertical axis represent the angle of the gate arm in Degree.

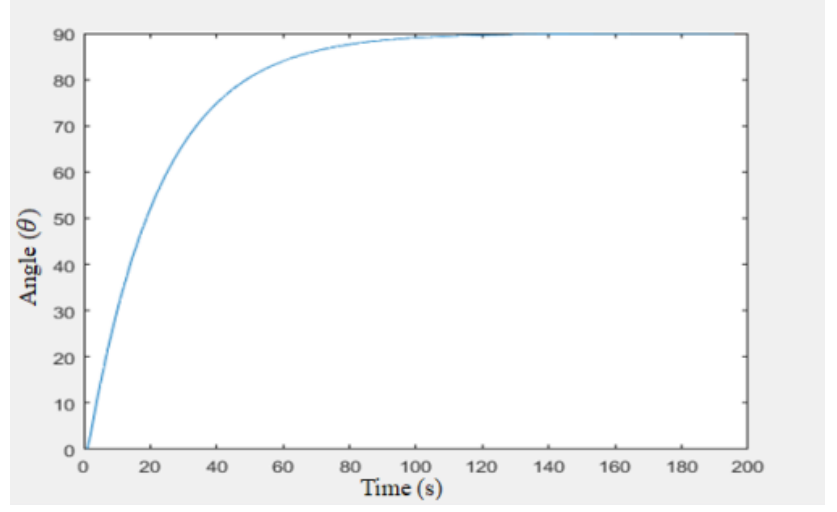

Fig.15 Simulation of the Step function for the desired motor characteristics 


\section{CONCLUSION}

Based on the piezoelectric crowd management system in this paper it can be conclude that this system will be useful in the crowded places, and it's an adaptive system in such manner that it can fit in any situation with a small modification in the geometry and the programming code, it's a new idea that can be applied without huge costs compare to the other system used in the market, However the cost of the actual system could be higher than the prototype presented in this paper, and that because this study is focusing on the system performance and accuracy in a small testing field quite more than the system materials quality, which can be easily improved according to the installation areas.

\section{REFERENCES}

[1] Jaffe, B,1971, Piezoelectric ceramics 1st edn, Academic Press .

[2] J. Keith Wing, E. Lamar, William L. Willis, Michelin Recherche et Technique SA France 1989, Revolution counter attached to tires, US4862486A.

[3] Ian Solomon, MotiGoldhirsh, Teva Pharmaceutical Industries Ltd 2011, US8539945B2.

[4] Michael O. Pekkarinen, Ludwig Wolf, Jr., Walker Woodworth, Baxter International Inc 1986, US4583975A.

[5] Sirohi J, Chopra I, 2000,'Fundamental Understanding of Piezoelectric Strain Sensors', Journal of Intelligent Material Systems and Structures, vol. 11, no. 4, pp. 246257.

[6] Takaaki Tsurumi, Tsutomu Sasaki, Hirofumi Kakemoto, 2004 ' Domain Contribution to Direct and Converse Piezoelectric Effects of PZT Ceramics', Japanese Journal of Applied Physics, Vol. 43, No. 11A, 2004, pp. 7618-7622.

[7] Howard V. Meredith 1944,'Human foot length from embryo to adult',Human Biology, Vol. 16, No 4. pp. 207-282.

[8] Levent Parali, Ali Sari, (2017), 'Vibration Modelling of Piezoelectric Actuator (PEA) Using Simulink Software', 4th International Conference on Electrical and Electronics Engineering. 